\title{
Análisis de publicaciones en promoción de la salud: una mirada a las tendencias relacionadas con prevención de la enfermedad ${ }^{*}$
}

\section{Analysis of Health Promotion Publications: A Look at Tendencies Related with Disease Prevention}

\section{Análise de publicações a promover saúde: um olhar às tendências relacionadas com prevenção das doenças}

Fecha de recepción: 30-10-14 Fecha de aceptación: 18-03-15 Disponible en línea: 30-06-15 doi: 10.11144/Javeriana.rgyps18-28.apps

Como citar este artículo:

Díaz-Mosquera SP, Rodríguez-Villamil LN, Valencia-González AM. Análisis de publicaciones en promoción de la salud: una mirada a las tendencias relacionadas con prevención de la enfermedad. Rev. Gerenc. Polít. Salud. 2015; 14(28): 32-47. http://dx.doi.org/10.11144/Javeriana.rgyps18-28.apps

Silvia Paulina Díaz-Mosquera**

Luz Natalia Rodríguez-Villamil***

Alejandra Milena Valencia-González****

Artículo de investigación, derivado del estudio Tendencias y aportes a la discusión de los trabajos de promoción de la salud en Colombia, publicados en revistas indexadas entre enero de 2000 y enero de 2010, realizado entre el 2010 y el 2012, acta no. ENFER04-2010 (feche de inicio: 23-10-2010, fecha de terminación: 23-05-2012), financiado por el Comité para el Desarrollo de la Investigación (CODI), de la Universidad de Antioquia.

** Odontóloga, magíster en Salud Colectiva, docente de la Facultad de Odontología de la Universidad de Antioquia, Medellín, Colombia. Correo electrónico: diazpaulina1@gmail.com.

**** Nutricionista dietista, magíster en Salud Colectiva, docente de la Escuela de Nutrición y Dietética de la Universidad de Antioquia, Medellín, Colombia. Correo electrónico:nataliarodriv@yahoo.com.

**** Enfermera, magíster en Salud Colectiva, docente de la Facultad de Enfermería de la Universidad de Antioquia, Medellín, Colombia. Autor de correspondencia. Dirección: Facultad de Enfermería, Universidad de Antioquia, Calle 64 No. 63-09, Medellín, Colombia. Teléfono: 574-2196332. Correo electrónico: alejandra.valencia@udea.edu.co. 


\section{Resumen}

Para acercarse al estado de la discusión sobre promoción de la salud en Colombia, se realizó una investigación cualitativa de tipo documental que analizó el contenido de artículos publicados en revistas indexadas entre el año 2000 y el 2010, con el objetivo de conocer las tendencias y los aportes a la discusión sobre promoción de la salud (PS). Una de las categorías relevantes fue la relación promoción de la salud-prevención de la enfermedad (PE), dentro de la cual se evidencian como tendencias: asumirlas como conceptos unificados, PS y PE como conceptos diferenciados y problematización de la PS en la práctica, que discute con el sistema de aseguramiento, con la interdisciplinariedad e intersectorialidad y los límites confusos entre PS y PE. Se plantea la necesidad de abordajes que superen la mirada dicotómica y disciplinar, así como reconocer en los escenarios cotidianos, lugares de concreción y aprendizaje de la PS.

Palabras clave: promoción de la salud; prevención de enfermedades; investigación; atención a la salud; condiciones sociales; Colombia

\section{Abstract}

We carried out a qualitative documental research in order to have a closer look at the current state of the discussion on health promotion in Colombia; our research analyzed the contents of articles published in indexed journals between the years 2000 and 2010. The purpose was to know the tendencies and contributions to the discussion on health promotion (PS). One of the relevant categories was the relationship between health promotion-disease prevention (PE), in which we can see tendencies such as: assuming both as unified concepts; PS and PE as different concepts; and problematization in practice of PS in, present in the discussion with insurance, interdisciplinarity, and 'intersectoriality', and the blurry borders between PS and PE. We raise the need for approaches that go beyond the dichotomic and disciplinary view, as well as recognition in everyday scenarios the places where Ps is learnt and realized.

Keywords: health promotion; disease prevention; research; attention to health; social conditions; Colombia

\section{Resumo}

Para se aproximar do estado da discussão sobre promoção de saúde na Colômbia realizou-se pesquisa qualitativa de tipo documental que analisou o conteúdo de artigos publicados em revistas indexadas entre o ano 2000 e 2010, com o objetivo de conhecer as tendências e aportes à discussão sobre promoção da saúde (PS). Uma das categorias relevantes foi a relação promoção de saúde-prevenção de doenças (PD), dentro da qual evidenciam-se como tendências: assumi-las como conceitos unificados, PS e PD como conceitos diferenciados e problematização da PS na prática, que discute com o sistema de asseguração, com a interdisciplinaridade e intersetorialidade e com os limites confusos entre PS e PD. Levanta-se a necessidade de abordagens a superar o olhar dicotómico e disciplinar, assim como reconhecer nos cenários cotidianos, lugares de concreção e aprendizagem da PS.

Palavras-chave: promoção de saúde; prevenção de doença; pesquisa; atendimento à saúde; condições sociais; Colômbia 


\section{Introducción}

Una de las discusiones vigentes en el campo de la salud tiene que ver con la manera como se articulan en la práctica los discursos sobre promoción de la salud (PS). Caben ahí las preguntas por los conceptos, nociones y teorías (1) que hacen parte del debate teórico práctico sobre este tema y que pueden enriquecer las acciones y propuestas en salud y la formación de los profesionales en el área. Es entonces cuando se plantea la pregunta acerca del estado de la discusión sobre Ps por parte de la comunidad académica en Colombia, discusión que da origen a esta investigación.

La Ps ha sido entendida desde diferentes perspectivas: como estrategia, campo de conocimiento, escenario de acción y reflexión, y como "práctica o corriente metodológica, o movimiento internacional" (2); los discursos sobre ella tienen diversos orígenes y en ellos se encuentran tendencias que van desde aquellas que enfatizan en las prácticas individuales y en los estilos de vida, hasta las que reclaman el compromiso intersectorial en pro del mejoramiento de las condiciones de vida de la población y que proponen retos para la acción, cuando se establecen relaciones entre la PS y la vida, la salud, la solidaridad, la equidad, la democracia y la participación, entre otras (3).

Estas tendencias dan cuenta de una mirada compleja de la PS, que en el caso implica reconocer que la salud no se limita a la enfermedad y a lo individual, ni tampoco es reducible al plano de los fenómenos observables, sino que propone una mirada a los procesos en constante movimiento (4), y, en consecuencia, su comprensión debe hacerse desde esta misma perspectiva, lo que implica cuestionar las maneras como se le mira en la teoría y en la práctica. Un ejemplo lo constituye su implementación en el contexto del actual Sistema General de Seguridad Social en Salud (sGSss), que ha configurado lógicas en las prácticas en salud, y entre estas, las que atañen a la Ps, que se alejan de sus postulados de transformación de las condiciones de vida $y$ en cambio enfatizan en acciones dirigidas a los individuos y centradas en la lógica de los estilos de vida (5).

En medio de esta realidad, cobra sentido reconocer la Ps como un concepto complejo, acogido por diversas disciplinas, nutrido por aportes de diferentes campos del conocimiento y todavía en construcción y cambio. Igualmente, se hace necesario reconocer la diversidad de tendencias y discursos sobre PS, como una posibilidad de avanzar en el debate teórico y a la vez identificar posibilidades de concreción en la práctica. Por lo anterior, el objetivo de la investigación fue analizar las tendencias y aportes a la discusión de los trabajos de promoción de la salud en Colombia, publicados en revistas indexadas desde enero del 2000 hasta enero del 2010.

El abordaje de esta temática tiene su importancia, como subraya Breilh (4), en que si bien el pensamiento científico no es la única forma de saber, sí dispone de características, herramientas y capacidades que le dan fuerza y poder. En este sentido, las construcciones académicas pueden convertirse en orientadoras de las tareas en salud dirigidas por los tomadores de decisiones.

En esta investigación emergieron tensiones entre la teoría y la práctica de la PS, y entre PS y PE, que dieron lugar a las categorías analíticas de la PS en el sGSss en nuestro país, los conceptos de salud que le subyacen y la presencia reiterada de la PE en relación con los discursos sobre Ps. El análisis de esta última categoría, en la cual se incorporan reflexiones sobre el pensamiento dicotómico, lo disciplinar y las interacciones entre teoría y prácticas, se presenta en este artículo. 


\section{Materiales y métodos}

\section{Tipo de estudio}

Se realizó una investigación cualitativa de carácter documental, la cual se caracteriza porque el investigador hace su trabajo sin participar directamente del mundo que estudia. Los documentos que se revisan, en el caso los artículos, pueden ser observados como ocurre con los hechos sociales (6), de tal forma que estos son el soporte material del fenómeno social por estudiar y por ello pueden entrevistarse mediante las preguntas que guían la investigación (7).

De esta manera, se construyó un diseño flexible que siguió un muestreo intencionado, en el cual, según Minayo (8), más que la cantidad importa la calidad y profundidad del objeto, en cuanto a los aportes y tendencias a la PS.

\section{Fuentes de información}

La estrategia de búsqueda planteó, en primer lugar, las fuentes de consulta, pues si bien existe diversidad de producciones sobre el tema, publicadas tanto en revistas científicas como en "literatura gris", se tomó la decisión de revisar la producción existente en revistas indexadas y se seleccionaron las bases de datos bibliográficas disponibles en ese momento en el Sistema de Bibliotecas de la Universidad de Antioquia: Pub Med, Science Direct, Lilacs, Willey Interscience, Ovid, Currents Contents, Springer Link, Scielo, MD Consult, Ebsco Medline y Jstor. Se buscaron aquellos artículos publicados entre el 2000 y el 2010, periodo que avanzaba en una década a la propuesta por Torres y Escobar (9) en su investigación sobre la discusión de la práctica en Ps entre 1991 y 1999, que dejaba un punto de partida para seguir en una línea ya explorada, pero en un campo no revisado, como lo fue el caso de los artículos publicados en revistas indexadas que abordaran el tema de la Ps en Colombia, y para ello se definieron como descriptores: promoción de la salud, health promotion $\mathrm{y}$ promoção da saúde.

De esta primera búsqueda, luego de descartar repeticiones, se obtuvieron 176 artículos, los cuales se leyeron para determinar su pertinencia; de esa manera, se descartaron aquellos que enunciaban el término promoción de la salud, pero no lo desarrollaban con profundidad en su contenido. Finalmente, para el análisis se seleccionaron 37 artículos que daban cuenta explícita de desarrollos de promoción de la salud.

Posteriormente, se clasificó y se valoró el material, se realizaron fichas de contenido y se elaboró una base de datos con la caracterización de la información consultada: descripción de las revistas, autores, instituciones editoras, ciudad y año de publicación, categorías centrales, objetivos y las relaciones con la PS. La lectura detallada de cada artículo permitió clasificarlos según el tipo de publicación y los temas tratados.

\section{Proceso de análisis}

Se hizo una lectura detallada de cada artículo, a modo de documentación, con propósito de análisis e interpretación (7), para extraer en fichas temas, tendencias y contradicciones. Tomando algunos elementos del análisis de contenido, se determinaron unidades de registro y análisis (6), en este caso de fragmentos de texto, teniendo presente su relación con las unidades de contexto (lugares concretos del texto en donde aparecen) (6). Estas unidades de registro se agruparon por categorías, con base en criterios semánticos (temas y áreas conceptuales) tales como: conceptos de PS, prácticas de PS, PS y sistema 
de salud, entre otras emergentes como fue el caso de la Promoción de la Salud y la Prevención de la Enfermedad —PS y PE-, la que por las tensiones que planteaba requería lecturas desde otras propuestas. Las categorías se ordenaron alrededor del objeto promocional que es la salud y, de esta manera, se elaboraron mapas que facilitaron la identificación de relaciones entre categorías como PS y salud, PS y entornos, PS y PE, entre otras, y se construyeron algunos memos empíricos, teóricos y analíticos (10), en los cuales se identificaron asuntos por profundizar en el análisis.

Posteriormente, se inició la escritura descriptiva y analítica de las principales categorías, para dar cuenta de las tendencias, entre ellas la relación entre PS y PE en la que se centra este escrito.

\section{Resultados}

Para dar cuenta de la caracterización general de la producción de artículos en promoción de la salud publicados entre el 2000 y el 2010, en la tabla 1 se detallan las revistas, su procedencia, la formación de los autores y los tipos de publicaciones.

Entre las revistas que publicaron en promoción de la salud se encuentran: Hacia la Promoción de la Salud, de la Universidad de Caldas (27,0\%); Facultad Nacional de Salud Pública, de la Universidad de Antioquia (16,2\%); Salud Pública, de la Universidad Nacional (13,5\%), e Investigación y Educación en Enfermería, de la Universidad de Antioquia (10,8\%); once revistas más publicaron uno o dos artículos en el periodo de referencia.

La procedencia institucional de las revistas 36 que se consultaron corresponde en $35,1 \%$ a la Universidad de Antioquia, 27,0\% a la Uni- versidad de Caldas, 21,6\% a la Universidad Nacional de Colombia y el $16,2 \%$ restante a otras universidades.

Más de la mitad de los autores $(65,7 \%)$ pertenecían al área de la salud, seguidos de $14,9 \%$ de profesionales de las ciencias sociales, $11,9 \%$ de estudiantes de pregrado, $\mathrm{y}$, finalmente, $7,5 \%$ de los artículos no daban cuenta de la formación de su autor.

Según tipo de publicación, $43 \%$ corresponden a investigaciones, $24 \%$ a ensayos, $24 \%$ a reflexiones y $8 \%$ a revisiones.

En cuanto a los artículos derivados de investigaciones, se encontró que desarrollan temas relacionados con: saberes y prácticas de niños, población en condiciones de exclusión social y profesionales de la salud $(11,14)$, estrategias y evaluación de programas de PS $(15,18)$ y su relación con la situación de salud $(19,20)$. También abordan asuntos de actividad física $(21,22)$, resiliencia (23), estilos de vida $(24,25)$ y sistema de salud $(26)$.

Los correspondientes a ensayos plantean visiones de los autores con respecto a las confusiones y diferencias entre PS y PE, el alcance de las acciones de PS (27-30), la crítica a los estilos de vida (31), algunas estrategias como la comunicación y el marketing sociales (32-34) y el partnership (35), así como críticas a la legislación en el sistema de salud (36). En los artículos de revisión se observó que las discusiones tienen elementos de evaluación (37) y hacen alusión a la importancia de la evidencia científica (38) y a la práctica profesional (39) en el marco de la Ps y la PE.

En la relación PS y PE como categoría de análisis, se identificaron tres tendencias que reflejan algunas de las preocupaciones, reflexiones y concepciones que expresan los autores: la PS y la PE como concepto unificado, la Ps y la PE como conceptos diferenciados 
Tabla 1. Caracterización de los artículos de Promoción de la Salud en Colombia publicados en revistas INDEXADAS ENTRE EL AÑO 2000 Y EL 2010

\begin{tabular}{|c|c|c|}
\hline Revista e institución de origen de los artículos & $\mathbf{N}^{\circ}$. & $\%$ \\
\hline Revista Hacia la Promoción de la Salud, Universidad de Caldas & 10 & 27,0 \\
\hline Revista de la Facultad Nacional de Salud Pública, Universidad de Antioquia & 6 & 16,2 \\
\hline Revista de Salud Pública, Universidad Nacional de Colombia & 5 & 13,5 \\
\hline Revista Investigación y Educación en Enfermería, Universidad de Antioquia & 4 & 10,8 \\
\hline Revista de la Facultad de Medicina, Universidad Nacional & 2 & 5,4 \\
\hline Avances en Enfermería, Universidad Nacional de Colombia & 1 & 2,7 \\
\hline Ciência \& Saúde Coletiva, Asociación Brasileña de Salud Colectiva (Abrasco) & 1 & 2,7 \\
\hline Colombia Médica, Universidad del Valle & 1 & 2,7 \\
\hline Revista Facultad de Medicina, Universidad Militar Nueva Granada & 1 & 2,7 \\
\hline Revista Gerencia y Políticas de Salud, Pontificia Universidad Javeriana & 1 & 2,7 \\
\hline Aquichan, Universidad de la Sabana & 1 & 2,7 \\
\hline Iatreia, Universidad de Antioquia & 1 & 2,7 \\
\hline Revista CES Odontología, Universidad CES & 1 & 2,7 \\
\hline Revista Facultad de Odontología, Universidad de Antioquia & 1 & 2,7 \\
\hline Perspectivas en Nutrición Humana, Universidad de Antioquia & 1 & 2,7 \\
\hline Total & 37 & 100,0 \\
\hline \multicolumn{3}{|l|}{ Número de autores por artículo } \\
\hline Entre 1 y 2 & 29 & 78,4 \\
\hline Entre 3 y 4 & 6 & 16,2 \\
\hline 5 y más & 2 & 5,4 \\
\hline Total & 37 & 100,0 \\
\hline \multicolumn{3}{|l|}{ Área de formación de los autores u ocupación } \\
\hline $\begin{array}{l}\text { Ciencias de la salud (enfermería, medicina, odontología, nutrición y dietética, educación } \\
\text { física, microbiología, fisioterapia, bacteriología, fonoaudiología y consultoría en salud } \\
\text { pública y promoción de la salud) }\end{array}$ & 44 & 65,7 \\
\hline Ciencias sociales (comunicación social, psicología, trabajo social) & 7 & 10,4 \\
\hline Profesores & 3 & 4,5 \\
\hline Estudiantes de pregrado & 8 & 11,9 \\
\hline Sin dato & 5 & 7,5 \\
\hline Total & 67 & 100 \\
\hline \multicolumn{3}{|l|}{ Tipo de artículo } \\
\hline De investigación & 16 & 43,2 \\
\hline Ensayo & 9 & 24,3 \\
\hline Reflexión & 9 & 24,3 \\
\hline De revisión & 9 & 8,1 \\
\hline Total & 37 & 100,0 \\
\hline
\end{tabular}

Fuente: elaboración propia 
y las reflexiones de los autores para hacer visibles las posibilidades de abordarlas.

\section{PS y PE como concepto unificado}

En algunos de los artículos se pudo evidenciar que los autores perciben la PS y la PE como un concepto unificado, lo que permite la identificación y análisis de elementos de complementariedad e importancia en el discurso sanitario nacional e internacional en relación con los modelos de atención en salud. Esta mirada tiene soporte, entre otros, en los análisis de autores como Silva, Herrera y Agudelo (26) y Suárez (36), sobre la normatividad e implementación de las políticas de salud en Colombia, en donde se hacen evidentes las limitaciones en la aplicación de la PS y la PE, lo que contrasta con los discursos curativos imperantes en el sistema.

En este sentido, Sanabria sostiene que los programas preventivos que buscan transformar los estilos de vida, constituyen un punto de convergencia para PS y PE como elemento de gran importancia en los procesos de formación de profesionales sanitarios, lo cual se hace explícito en la práctica: "[...] intervenir los estilos de vida se convierte en una de las acciones más eficaces para la prevención de la enfermedad y la promoción de la salud [...] de tal forma que cada vez es más frecuente que los médicos recomienden a sus pacientes la modificación de ciertas conductas que atentan contra su salud" (25).

En los artículos analizados se afirma que este abordaje de la Ps y la PE como concepto unificado, hizo que se incorporara al lenguaje de los actores de las instituciones de salud la expresión "P y P", lo que evidencia una mirada unificadora e indiferenciada en la cual aparecen las dos como partes necesarias de un mismo término. Además, con el auge de la Atención Primaria en Salud, en la cual la PS y la PE se consideran indispensables, se ha reforzado el abordaje complementario de ambos conceptos, pero también se ha llevado al desdibujamiento de las particularidades de la Ps, por el énfasis que se pone sobre las acciones orientadas al autocuidado y, en general, sobre la prevención.

Eslava plantea que aun cuando existe un reconocimiento de la Ps como posibilidad para trascender la mirada biomédica de los procesos salud-enfermedad-atención, su mención y análisis conjunto hacen que la PS aparezca en referencia a lo preventivo, como dimensión contenida en ella o como complemento: "[...] tiene que ver con una confusión y cierta sutil rivalidad que se ha generado entre promoción y prevención lo que ha llevado a que se sobrepongan acciones, se generen suspicacias y, en últimas, se impida el despliegue de estas dos dimensiones del accionar en salud" (27).

\section{PS Y PE como conceptos diferenciados}

Cuando se mencionaron la PS y la PE como conceptos separados, los autores sustentaron la existencia de génesis, significados, objetivos, objetos de intervención y modelos teóricos que los distinguen.

Así, se hizo alusión a la importancia de entender la Ps a partir de la mención reiterada de múltiples definiciones y enfoques que existen sobre ella, según aportes de organismos y autores como Henry Sigerist, quien la introdujo a mediados del siglo xx como una de las tareas fundamentales de la medicina (40), la perspectiva de integración en el Informe Lalonde de 1974 (41) y la propuesta de Salud para Todos del año 2000 (42), como premisa de Alma Ata, que posteriormente se retoma como punto central de la Carta de Ottawa y sus cinco áreas estratégicas: políticas públicas saludables, creación de ambientes favorables, reforzamiento de la acción comunitaria, desarrollo de aptitudes 
personales y reorientación de los servicios de salud (43), así como en las declaraciones sobre PS y las prioridades de la oms en la Quincuagésima Primera Asamblea Mundial de Salud, celebrada en 1998 (44), para el trabajo en Ps: promover la responsabilidad social en salud, aumentar la capacidad de las comunidades y habilitar al individuo, ampliar y consolidar las alianzas estratégicas, aumentar las inversiones en el desarrollo sanitario, garantizar la infraestructura necesaria para la PS. Todos estos son referentes indiscutibles, a través de los cuales la ps ha ganado el estatus de política de salud pública a nivel internacional, como lo afirma Prieto: "más marcada por la discusión política, económica y social del problema de la salud; ejemplo de ello son las conferencias, cartas y declaraciones que los países han formulado en los últimos tiempos" (39).

Dentro de esta mirada de la Ps como concepto diferenciado se encontraron varias posturas, entre las que se destacan: la PS como estrategia y política sanitaria y la PS como proceso de transformación social, política y económica.

En la mención de Ps como estrategia y política sanitaria, desarrollada en correspondencia directa con los servicios de salud y en el contexto del sGsss se destacan las actividades, procedimientos, cumplimiento de guías de atención y planes de beneficios en el ámbito individual y colectivo, en los que el aspecto predominante es el énfasis en el fomento de estilos de vida saludables y el control de los individuos sobre la propia vida. Además, se identificaron acciones extramurales tendientes a reforzar habilidades y competencias a través de la información sobre los factores de riesgo y de protección, derechos y deberes de los pacientes y actividades de educación para la salud a nivel individual, familiar y comunitario. En este orden de ideas, se encontró que algunos de los artículos dan cuenta de las críticas que se formulan a los estilos de vida, como responsabilidad exclusiva de los individuos, en desconexión con aspectos sociales, económicos y culturales más estructurados. En cuanto a la concreción de la Ps en el marco del SGSss, las críticas se relacionan con la insuficiencia de recursos financieros, la incapacidad de establecer alianzas intersectoriales, el predominio de la curación en el modelo de salud, la fragmentación de acciones y actores, la baja participación de la comunidad y la poca acción estatal como ente regulador. En este sentido, Hernández afirma:

En mucho, lo que haga el sector tanto en el espacio individual como en el colectivo de las acciones de promoción de la salud, dependerá de las reglas de juego del sistema, de la inclusión de las actividades de promoción en los beneficios que deben asegurase a la población y de la capacidad de agencia y habilidad desarrollada por el sector para actuar en los planos individual y colectivo. Una evaluación de las funciones esenciales del Sistema de seguridad social en Colombia mostró a la promoción de la salud como la función peor ubicada por asignación insuficiente de recursos financieros, la incapacidad de convocatoria y liderazgo de las autoridades sanitarias para desarrollar alianzas con otros sectores, la insuficiente reorientación del sector hacia un modelo de atención con mayor énfasis en la atención primaria, y porque las responsabilidades de acción aparecen fragmentadas y diluidas en múltiples planes y actores. (20)

Ahora bien, la Ps entendida como proceso de transformación social, política y económica, se sitúa en el contexto de la salud como derecho; desde allí, busca unas condiciones favorables para el desarrollo de la salud y la vida con dignidad, mediante un trabajo por la equidad y la justicia social. En este sentido, los autores señalan las dificultades para la concreción de esta manera de concebir la 
PS, en medio de las políticas sociales neoliberales que han influido en las reformas de salud desde la lógica del mercado, como lo expresan Gómez y González:

El informe del Banco Mundial, cuyo contenido ha ejercido una profunda influencia sobre la política social en todo el mundo desde fines del siglo xx, se preocupa por la pobreza pero no por sus causas; supone las desigualdades como datos, y propone acciones para reducir los efectos de la inequidad, no para controlar su estructura causal. Si se examinan en detalle, las políticas impuestas por el Banco Mundial y el Fondo Monetario Internacional contradicen en la teoría y en la práctica los planteamientos de las conferencias internacionales sobre promoción de la salud que resaltaban la responsabilidad del Estado frente a la reducción de las inequidades sociales, la democratización de las decisiones y la protección del ambiente. (37)

Una de las propuestas para abordar la PS como proceso de transformación social la sustenta García (29), basado en Franco, como una realidad cotidiana inmersa en el concepto de proceso vital humano, en el cual se supera la oposición salud-enfermedad y ambas se comprenden como parte dialógica de la vida. Adicionalmente, en varios de los artículos consultados, la Ps tuvo un nexo con nociones como bienestar, empoderamiento, satisfacción, educación, participación, comunicación, calidad de vida y determinantes. Esta última, ligada a los discursos de las declaraciones en Ps como posibilidad de revisar aspectos relacionados con la salud que no se limiten a los servicios asistenciales. En cuanto a la participación, esta se considera como una estrategia clave para hacer posible la Ps, a la cual se vincula bien sea como participación social, o bien como participación comunitaria y, además, es entendida como propuesta para que las personas tomen con- trol sobre su propia salud, como se propuso en Ottawa (43).

A partir de los argumentos de algunos autores acerca de la PS y PE como conceptos diferenciados, se devela un claro afán por hacerlas posibles y cotidianas en las acciones de salud. Así, se identifica a la PE como concepto previo a la Ps y heredera de la comprensión de la enfermedad desde las teorías contagionista, miasmática, comportamental, higiénica y religiosa, y de la fuerza que adquirieron las propuestas de la historia natural de la enfermedad y los niveles de prevención. De aquí se desprende una razón para comprender la importancia y el protagonismo de las acciones de PE en los discursos y prácticas.

La PE se vincula principalmente a la epidemiología tradicional, la cual, desde la perspectiva de la causalidad, ha posicionado con fuerza lo relacionado con la identificación y control de factores de riesgo de individuos o grupos específicos, la reducción de gastos en salud como prioridad, los resultados en el corto o mediano plazo y el fomento de estilos de vida saludables. Este último, por su proximidad al componente curativo, ha estado muy cercano a las prácticas sanitarias y a la normatividad que las regula, como por ejemplo la Resolución 412 del 2000 (45).

Pese a que la prevención se plantea en dirección a los individuos y a grupos sociales, se percibe la carencia de un enfoque integral, toda vez que en la práctica predominan las acciones individuales, las cuales son más visibles en el momento de medir y costear las acciones realizadas tanto en el régimen contributivo como en el subsidiado. Lo anterior tiene que ver exclusivamente con las acciones de carácter vertical direccionadas por los profesionales de la salud, dejando apenas enunciada o muy incipiente la participación comunitaria. 
Autores como Eslava (27), Prieto (39), Gómez y González (37) aportan algunas discusiones sobre las dificultades de la lógica de la causalidad para reconocer el factor que es preciso intervenir, puesto que hay una tendencia a reducir a factores de riesgo aquellas condiciones sociales, económicas y políticas que determinan en buena medida los procesos de salud-enfermedad y que demandan otra manera de asumir la prevención desde la medicina social, en la cual la enfermedad se reconoce en su contexto histórico, social y político, en donde a la vez se dan procesos protectores y también procesos que deterioran los modos de vida.

\section{Reflexiones sobre la relación PS-PE}

En relación con el panorama estudiado, existe una apuesta de los autores por la búsqueda de claridad epistémica, teórica y metodológica, acerca de PS y PE, que más allá de contraponerlas, permita problematizar y recrear las discusiones en la práctica.

Los autores reiteran como reto la posibilidad de promover la salud en un sistema de aseguramiento centrado en la atención de enfermedades, autocuidado y autogestión, y con poco impacto de sus acciones, que hace más visible el contexto de iniquidades en las condiciones de vida de los colombianos. Así, que más que un asunto de decisión técnica, lo analizan como una dificultad teórica y práctica que sería posible afrontar a partir de un compromiso ético, político y de coherencia con las realidades colombianas. Realpe (30) y Prieto (39) ejemplifican lo anterior cuando afirman:

[...] para conseguirla [la Ps] se requiere de ciertas condiciones de vida, como son: la paz, la educación, la vivienda, la alimentación, la renta, la justicia social, la equidad y un ecosistema estable, podemos deducir que son precisamente estas condiciones, las que le hacen falta a la mayoría de los colombianos, por lo tanto la PS se convierte en una utopía en tanto cada sector, al cual le compete la responsabilidad, no modifique favorablemente dichas condiciones. (30)

Las estrategias de promoción y prevención en salud, forman parte o son producto de este desarrollo teórico e investigativo, en el cual parece ser que la velocidad de producción teórica sobre el tema, supera la capacidad de respuesta política de los pueblos. (39)

Otro aspecto de importancia es el que tiene que ver con los actores de la Ps y la PE, pues si bien en los artículos se destaca el papel del sector salud y sus profesionales, se reconoce la necesidad de fortalecer las acciones interdisciplinar, interinstitucional e intersectorial como condiciones para hacer realidad una PS y una PE que trasciendan las actividades educativas desarticuladas de los contextos, para que más allá de tener "un listado de buenas intenciones" (30), se conviertan en vivencia cotidiana en términos de una vida digna. En este sentido, se afirma:

Un desarrollo exitoso de la promoción de la salud y la prevención de la enfermedad en la cual estén comprometidos todos los actores, podría marcar la diferencia en la equidad, accesibilidad, calidad, eficiencia, efectividad y economía del sistema de salud. (35)

Sin embargo, es importante enfatizar que la promoción de la salud y la prevención de la enfermedad se constituyen en procesos que no solo les competen a los profesionales del área de la salud, sino que le competen prioritariamente al Estado, dado el papel que le asiste para generar estrategias de trabajo intersectorial, interinstitucional e interdisciplinario de una manera sistemática, deliberada y regulada. (12)

Por último, los autores plantean la confusión que existe en los límites conceptuales entre Ps 
y PE, como una dificultad para la formación de profesionales, pues la heterogeneidad y complejidad de los discursos conduce a prácticas que sobreponen las acciones asociadas a estos conceptos, y las estrategias para su vivencia efectiva son acogidas según la disposición o importancia que cada profesional les dé como acciones preventivas o promocionales, entendidas desde los factores de riesgo, por un lado, y desde un carácter social y político, por otro. Se encuentran, entonces, autores que apuestan por una diferenciación que permita la calidad en las acciones de cada una, es decir, una PE contextualizada, intencionada y efectiva en la reducción de las enfermedades, y otros que apuestan por la búsqueda de una ps que, desde las políticas de salud, permita unas mejores condiciones de vida:

Los retos que plantea la ps van desde la misma conceptualización de la Ps, como realidad discursiva y escenario de práctica específica, hasta el despliegue de un efectivo trabajo interdisciplinario que posibilite la construcción de ambientes y estilos de vida saludables a la vez que acompañe procesos de organización social y lucha política. (27)

\section{Discusión}

Los artículos analizados muestran una búsqueda de los autores por hacer posibles la PS y la PE en medio de los retos relacionados con el sGsss y de las condiciones de iniquidad en el contexto colombiano. En esta búsqueda se hace presente la necesidad de una relación permanente teoría-práctica, como soporte para transformar las realidades a partir de dos elementos de análisis interdependientes. El primero tiene que ver con las oportunidades y/o dificultades que ofrece la tendencia a unir o separar las reflexiones sobre PS y PE. El segundo está asociado al predominio del trabajo disciplinar y sectorial para abordarlas.
En la tendencia a separar o unificar los conceptos subyace una clara intención de los autores por encontrar una manera de comprender la PS y la PE a partir de todo el acervo de experiencias, discursos y declaraciones que las han hecho visibles. Lo anterior aporta al análisis de su complementariedad cuando se unifican y cuando se separan para resaltar el aporte y valor de cada una. Este proceso de discusión conceptual muestra aspectos de una lógica dicotómica para construir conocimiento, que se expresa en cuatro propiedades desarrolladas por García (46), aplicables a la discusión sobre PS y PE, como se explica a continuación.

El binarismo como percepción del mundo en pares conceptuales: la PS de la salud como buena, la PE como mala; la oposición, como una legitimación de una tensión o conflicto: la PS versus la PE; la subordinación, determinada por el pensamiento hegemónico: la PE subordina a la PS en su posición como elemento de la estructura de dominio del paradigma morbicéntrico en la que ambas están presentes (РуP), pero en la práctica la promoción se diluye; la generalización y exclusión negativa, cuando el elemento que se subordina se presenta peyorativamente como negación de la instancia subordinante, es decir, se presenta la Ps como superior, como lenguaje positivo de la vida y la $\mathrm{PE}$ como alusión a lo negativo: la enfermedad.

Lo anterior puede dificultar la comunicación, en términos de lo que entendemos y pretendemos con las acciones preventivas y promocionales, dejando en segundo plano lo que requieren los fenómenos o problemas que encontramos en las realidades y lo que representa un reto para reconocer las particularidades de PS y PE en las necesidades poblacionales, sin acudir a la negación para hacerlas visibles y posibles. En este sentido, se propone atender los planteamientos de Maldonado sobre la salud y la enfermedad, 
cuando señala la ausencia de conflicto entre ellas y se asumen los modos en que "se potencia - o se dejan de potenciar-acciones, decisiones y relaciones que contribuyen a que la vida misma tenga horizontes mejores, más amplios y más profundos" (47), o de Almeida, cuando propone considerar al tiempo unidad y diversidad, complementariedades y antagonismos como posibilidad para “crear síntesis y operar tránsitos [...] entre los saberes de la vida y los conocimientos de la ciencia" (48), propuesta que permite reconocer las limitaciones del pensamiento dicotómico sobre las realidades concretas.

El segundo elemento que emerge al analizar la producción científica en Colombia con respecto a la PS y PE, tiene que ver con las dificultades para el trabajo interdisciplinar e intersectorial, presentes en la primacía del sector salud y sus profesionales para orientarlas. Aquí es importante reconocer los innegables aportes de las disciplinas (49), como categorías organizadoras del conocimiento científico en términos de los avances tecnológicos, que en el sector de la salud han permitido mejores diagnósticos y propuestas de tratamiento. Sin embargo, como se evidencia en los resultados de esta investigación, la tradición de formar profesionales de la salud desde un enfoque principalmente biologicista y técnico, nos enfrenta a dificultades para la comprensión e integración con las realidades con que nos relacionamos.

Ambos elementos, la relación teoría-práctica y la intersectorialidad e interdisciplinariedad en relación con la PS, fueron contemplados en el trabajo realizado por Torres y Escobar (9), cuando se refieren a la primera como una dificultad relacionada con la división del trabajo: "los que piensan, los que deciden y los que hacen", y la segunda en alusión a las dificultades en su operacionalización, en correspondencia con la falta de claridad sobre los sectores llamados a interactuar y las dificultades para trabajar en articulación con otros.

Por su parte, Robledo y Agudelo (50) en su análisis teórico sobre las definiciones de PS, identifican que en su fase de consolidación, comprendida entre 1987 y 2008, la intersectorialidad aparece en relación con la acción articulada del Estado y la sociedad en una dinámica en la que son los expertos y los servicios de salud quienes coordinan.

Se encuentran también análisis sobre la PS en Colombia como el de Mantilla (51), quién además de señalar la "inconveniente confusión" entre PS y PE, invita a la búsqueda de acciones de PS que involucren sectores diferentes al de salud y nociones lejanas a la ausencia de enfermedad, para apuntar a una transformación de las condiciones de salud.

Los hallazgos de la presente investigación y lo encontrado por otros autores ponen en el panorama de la Ps en Colombia la limitación creciente y constante del pensamiento dicotómico y la hegemonía disciplinar que caracterizan el conocimiento y aplicación de la PS y la PE. Así, es posible confirmar que no es desde el campo de la salud de manera aislada, ni solo desde el análisis teórico, como se fortalece la praxis de ambas. De esta manera, se puede acoger la potencialidad de las teorías y las prácticas como: "la forma para recuperar la historicidad de lo dado, con todas las posibilidades que contenga, según sea la constelación de sujetos concurrentes en la situación social" (52). En ello existe la posibilidad de reconstruir prácticas a partir de las necesidades de los sujetos, y de la especificidad de los fenómenos para integrar la historia como experiencia del presente, en la que se desplieguen las direcciones posibles para abordar, en este caso, las condiciones del proceso vital humano en contextos particulares. Asimismo, Zemelman (52) invita a que, como investigadores, nos 
comprometamos, más allá de la tecnología investigativa que limita el pensamiento a la razón instrumental, para lo cual es necesario ubicarnos frente a las realidades, preguntándonos por las diferentes maneras en que pueden nombrarse y articularse los fenómenos, en términos de las alternativas de potenciación, de lo no significado, de la necesidad de sentido y de ampliación de subjetividad.

Podemos entonces referirnos a la ps y a la PE desde sus diferencias, como proponen algunos autores en esta investigación, pero también es necesario ubicarlas en relación con su afectación mutua (no fusión), considerándolas como dispositivos que potencian la vida y como conceptos universales en la búsqueda de bienestar de los seres humanos, pero que no son posibles como arquetipos, porque su construcción e importancia solo tiene sentido para cada territorio y a partir de lo que representan los procesos vitales en la cotidianidad de los seres humanos, pues es allí donde existen, crean, realizan, proponen y coexisten diversas formas de conocimiento elaboradas a partir de la historia, la tradición y la experiencia, en una comprensión de la salud en su carácter histórico, multidimensional y dialéctico (2).

En consecuencia, como comunidad académica, corresponde visibilizar esas prácticas que los sujetos y los colectivos generan en su vida concreta, con aportes a sus procesos de bien vivir, y dar cuenta de que las acciones desarrolladas en diversos niveles y por distintos agentes - no solo en el sector salud- pueden estar planteadas como PE, o como PS, pero finalmente, conllevan diversas dinámicas, que no necesariamente se pueden segregar. Por ejemplo, lo que puede generarse en una comunidad que ha carecido de agua potable cuando se dota del servicio, cuando una persona que ha vivido en la exclusión accede a la educación, o cuando en una comunidad se recuperan saberes tradicionales o se reivindican derechos; ninguno de estos casos podría clasificarse como acciones que previenen enfermedades o promueven la salud, pues, en definitiva, se suscitan dinámicas diversas que apuntan a dignificar la vida.

En últimas, más que clasificar acciones en preventivas o promocionales o de su unificación, se trata de reconocer las posibilidades y limitaciones de unas y otras, de divulgar experiencias dentro y fuera del ámbito institucional, como plantea Granda cuando afirma que se requiere "interpretar las acciones vitales naturales y sociales diversas, aprender de ellas para organizar propuestas de cuidado de la vida natural y social mediando con la ciencia, la economía y la política con miras a impulsar la salud poblacional" (53), asunto que representa un reto complejo, pues implica que los profesionales de la salud, en lugar de acudir a enseñar, tomen el papel de aprendices, al integrar y aprender de aquellas personas e instituciones que, intencionalmente o no, y desde cualquier sector o disciplina, construyen vivencias que promueven la salud, al margen de los procesos investigativos formales o de la lógica del conocimiento indexado para nutrir la teoría a partir de las prácticas. A este respecto, “es necesario comprender a los seres humanos como sujetos éticos capaces de construir conocimiento independientemente de los expertos o de las instituciones y con base en ese conocimiento, dar significado, valor y sentido a su mundo y práctica” (54).

Es preciso asumir, como plantea Ghiso (55), que entre teoría y práctica existe una relación necesaria y contradictoria, porque en las prácticas sociales se construye conocimiento de manera crítica a partir de las experiencias de sujetos sociales que actúan en una realidad sobre la cual se constituyen y a la cual aportan, incorporando los elementos de cada disciplina 
para comprender la realidad, pero también "revalorizando el diálogo, el intercambio y la combinación entre diversos saberes" (56).

Surge entonces la necesidad de resignificar el actuar de los profesionales y la formación disciplinar, con el fin de "desarrollar la capacidad para 'inter-actuar' en la diferencia y creer honestamente que la verdad es plural y la diversidad una fuente inagotable de riqueza" (57), con lo que se podrían potenciar espacios de discusión que superen los cuadros comparativos de PS y PE, las aulas de clase como lugar central para el aprendizaje, y así avanzar hacia confrontaciones reflexivas con las realidades cotidianas, admitiendo que "el conocimiento es configuración de mundo" (58), permitiendo que los procesos que promueven la salud y previenen la enfermedad se vivan y construyan más allá del protagonismo de una disciplina o del sector salud.

Asimismo, podemos acompañar el diseño de los diagnósticos sobre las condiciones de vida, reconociendo el capital social propio de diferentes cosmovisiones que se abren campo en los discursos y prácticas, lo que requiere "tomar en cuenta, además de la norma técnica, la idea que los diversos tienen acerca de lo que es el 'buen vivir' y 'la felicidad" (59), lo cual incluye, pero no centra las posibilidades de la Ps en el sector salud o en la Academia; contrariamente a ello, abre el camino a lo que Sousa Santos desarrolla como la ecología de saberes, que invita a "aprender de otros conocimientos"(60), con los cuales, en últimas, las personas toman decisiones, resuelven problemas y construyen la vida, en medio de la invisibilización causada por el pensamiento hegemónico.

\section{Conclusiones}

La discusión sobre Ps en Colombia apunta a una búsqueda de acciones por una vida digna que requiere el concurso de diferentes actores, así como el abordaje de las relaciones sectoriales y disciplinares que han sido ampliamente descritas por este y otros trabajos. Es claro que las dinámicas institucionales relacionadas principalmente con la productividad, las jerarquías y los objetos disciplinares se dan en una estructura en la cual se dificulta la concreción de lo "inter" y en esta búsqueda de cómo hacerlo, proponemos iniciar o fortalecer en los pequeños grupos (sean estos académicos, comunitarios, etc.), una reflexión sobre la manera en la que podemos interactuar para promocionar la salud a partir de crear, de manera cotidiana, espacios de aprendizaje hacia el trabajo conjunto en la diversidad, de modo que se desestructuren las relaciones y sea posible aprender otros lenguajes y miradas en el mundo; es decir, que las prácticas y los contextos hablen primero, de modo que las acciones sean pertinentes y propicien la construcción de hechos intersectoriales e interdisciplinarios que nos ayuden a comprender el potencial de las sinergias en lo que hacemos y teorizamos, acercándonos a las realidades con miradas creativas y complejas.

\section{Referencias bibliográficas}

1. Minayo MCS. La artesanía de la investigación cualitativa. Buenos Aires: Lugar; 2009.

2. Chapela MC, Jarillo E. Promoción de la salud, siete tesis del debate. Cuad Méd Soc (Ros). 2001; 79: 59-69.

3. Marchiori P. Una introducción al concepto de promoción de la salud. En: Czeresnia D, Machado C, organizadores. Promoción de la salud. Conceptos, reflexiones, tendencias. Buenos Aires: Lugar; 2006. pp. 23-24.

4. Breilh J. Epidemiología crítica. Ciencia emancipadora e interculturalidad. Buenos Aires: Lugar; 2003.

5. Rodríguez N, Valencia A, Díaz P. Promoción de la salud y sistema de salud colombiano: Reflexiones sobre la formación de profesionales. Hacia Promoc Salud. 2013; 18 (1): 26-40.

6. Galeano E. Estrategias de investigación social cualitativa. El giro en la mirada. Medellín: La Carreta; 2004.

7. Valles M. La investigación documental: técnicas de lectura y documentación. En: Técnicas cualitativas de 
investigación social: Reflexión metodológica y práctica profesional. Madrid: Síntesis; 1997. pp. 109-39.

8. Minayo MC. Ciencia, técnica y arte: el desafío de la investigación social. En: Investigación social. Teoría, método y creatividad. Buenos Aires: Lugar; 2003.

9. Torres B, Escobar M. El estado de la discusión y de la práctica en promoción de la salud en Colombia 1991-1999 [tesis de magíster en Salud Colectiva]. Medellín: Universidad de Antioquia, Facultad de Enfermería; 2001.

10. Flick U. Introducción a la investigación cualitativa. Madrid: Morata; 2007.

11. Díaz M, Jaramillo G. La promoción de la salud en el ámbito escolar del colegio José Celestino Mutis. Rev Fac Odontol Uni. Antioq. 2005; 17 (1): 34-42.

12. Ríos K, Duarte J, Mesa L, Zapata J, Palacio S. Nociones y experiencias en promoción de la salud y prevención de la enfermedad de bacteriólogos y laboratoristas clínicos egresados de la Universidad de Antioquia. Rev Fac Nac Salud Pública. 2005; 23 (1): 19-31.

13. Rodríguez LN, Arias B. La alfabetización de adultos: escenario potencial para la promoción de la salud. Invest Educ Enferm. 2009; 27 (2): 236-42.

14. Ospina B, Sandoval J, Aristizábal C, Ramírez M. La escala de Likert en la valoración de los conocimientos y las actitudes de los profesionales de enfermería en el cuidado de la salud: Antioquia, 2003. Invest Educ. Enferm. 2005; 23 (1): 14-29.

15. González M, Jaramillo C. Modelo de evaluación para los programas de promoción de la salud desarrollados por las instituciones prestadoras de servicios de salud - IPS, Ley 100 de 1993, Manizales 2000. Hacia Promoc. Salud. 2001; 6: 53-69.

16. Gutiérrez A, Gómez O. Process Evaluation of the Healthy Schools Strategy in the Urban Area in Cali, Colombia. Colomb Med. 2007; 38 (4): 386-94.

17. Hoyos J, Duvaltier I, Giraldo W. Límites de los programas de promoción y prevención: Una perspectiva psicoanalítica. Iatreia. 2003; 16 (2): 132-9.

18. Salazar L, Díaz C. La evaluación-sistematización: una propuesta metodológica para la evaluación en promoción de la salud. Un estudio de caso en Cali, Colombia. Ciênc Saúde Coletiva. 2004; 9 (3): 545-55.

19. Agudelo A, Zapata D, Martínez E. La dinámica familiar y el fomento de la salud bucal en poblaciones desplazadas y destechadas. CES Odontol. 2007; 20 (2): 23-30.

20. Hernández A. Lineamientos para el desarrollo de una estrategia de intervención en atención primaria en salud y promoción de la salud para la población desplazada, pobre y vulnerable de la zona sur oriental de Cartagena. Rev Gerenc Polít Salud. 2003; 2 (5): 78-106.

21. Martínez E, Saldarriaga J, Sepúlveda F. Actividad física en Medellín: desafío para la promoción de la salud. Rev Fac Nac Salud Pública. 2008; 26 (2): 118-22.

46 22. Prieto A. Modelo de promoción de la salud, con énfasis en actividad física, para una comunidad estudiantil universitaria. Rev Salud Pública. 2003; 5 (3): 284-300.

23. Ospina D, Jaramillo D, Uribe Vélez T. La resiliencia en la promoción de la salud de las mujeres. Invest Educ Enferm. 2005; 23 (1): 78-89.

24. Morales R, Restrepo S. Programas de restaurantes comunitarios. Un proceso dinamizador de la promoción de la salud. Municipio de Caldas Antioquia. Perspect Nutr Hum. 2005 (13): 31-45.

25. Sanabria-Ferrand P, González L, Urrego D. Estilos de vida saludable en profesionales de la salud colombianos. Estudio exploratorio. Rev Fac Med 2007; 15 (2): 207-17.

26. Silva L, Herrera V, Agudelo C. Promoción, prevención, municipalización y aseguramiento en salud, en siete municipios. Rev Salud Pública. 2002; 4 (1): 36-58.

27. Eslava-Castañeda JC. Promoción y prevención en el sistema de salud de Colombia. Rev Salud Pública. 2002; 4 (1): 1-12.

28. Eslava-Castañeda JC. Rethinking How Health is Promoted in the Colombian General HealthRelated Social Security System. Rev Salud Pública. 2006; 8 (Supl 2): 106-15.

29. García C, Tobón O. Promoción de la salud, prevención de la enfermedad, Atención Primaria en Salud y Plan de Atención Básica. ¿Qué los acerca? ¿Qué los separa? Hacia Promc. Salud. 2000; 5: 7-21.

30. Realpe C. La promoción y la prevención en el sistema de salud Colombiano. Hacia Promoc. Salud. 2002; 7: 7-12.

31. Martínez O. La promoción de la salud. Némesis médica. Rev Fac Med. 2003; 51 (3): 158-63.

32. Forero JA. La comunicación social para la promoción de la salud. Hacia Promoc Salud. 2003; 8: 59-75.

33. Forero JA. El mix de las comunicaciones: comunicación total en la promoción de la salud. Hacia Promoc Salud. 2004; 9: 53-75.

34. Forero Santos JA. El marketing social como estrategia para la promoción de la salud. La Sociología en sus Escenarios. 2009; (20): 1-20 [Internet] [acceso: 10 de abril del 2014]. Disponible en: http:// aprendeenlinea.udea.edu.co/revistas/index.php/ ceo/article/view/7021/6440.

35. Molina G. Partnership: una estrategia de asociación para fortalecer la promoción de la salud y la prevención de enfermedades a escala municipal. Rev Fac Nac Salud Pública. 2001; 19 (2): 75-88.

36. Suárez EM. Promoción de la salud y prevención de la enfermedad. Comentarios a la Ley 715 de 2001. Aquichan. 2003; 3 (1): 9-15.

37. Gómez R, González E. Evaluación de la prevención de la enfermedad y la promoción de la salud: factores que deben considerarse. Rev Fac Nac Salud Pública. 2004; 22 (1): 87-106.

38. Buendía J, Sánchez J. Using Systematic Reviews for Evidence-Based Health Promotion: Basic Methodology Issues. Rev Salud Pública. 2006; 8 (Supl. 2): 94-105.

39. Prieto A. Promoción de la salud y prevención de la enfermedad, desde la Fisioterapia: Revisión 
conceptual. Rev Fac Med Univ Nac Colomb. 2004; 52 (1): 62-74.

40. Terris M. Conceptos de la promoción de la salud: Dualidades de la salud pública. En: Promoción de la Salud: Una antología. Organización Panamericana de la Salud. Publicación Científica; 557, Washington, D.C: ops; 1996. pp. 37-38.

41. Lalonde M. A New Perspective on the Health of Canadians. A Working Document. Ottawa: Minister of National Health and Welfare Canada; 1974.

42. Organización Mundial de la Salud. Estrategia mundial de salud para todos en el año 2000. Serie Salud Para Todos. No. 3. Ginebra: oms, 1981.

43. Organización Mundial de la Salud. Carta de Ottawa para la Promoción de la Salud. En: Primera Conferencia Internacional sobre la Promoción de la Salud; Ottawa, Canadá. Geneva; 1986.

44. Organización Mundial de la Salud. $51^{\circ}$ Asamblea Mundial de la Salud. WHA51.12 Punto 20 del orden del día 16 de mayo de 1998. Promoción de la Salud. Washington, 1998.

45. Colombia. Ministerio de Salud. Resolución 412 de febrero 25 de 2000. Por la cual se establecen las actividades, procedimientos e intervenciones de demanda inducida y obligatorio cumplimiento y se adoptan las normas técnicas y guías de atención para el desarrollo de las acciones de protección específica y detección temprana y la atención de enfermedades de interés en salud pública.

46. García A. La descolonización de los saberes. Itinerarios de paraconsistencia. 20 Años del Capítulo Español de isko. Actas del X Congreso Isko Capítulo Español. Universidad da Coruña; 2012 [Internet] [acceso: 10 de julio del 2014]. Disponible en: http://ruc.udc.es/dspace/bitstream/2183/11629/1/ CC_132_art_2.pdf.

47. Maldonado CE. La complejidad de la salud. Interacciones entre lo biológico y lo social. En: Morales MC, editora. Repensando la naturaleza social de la salud en las sociedades contemporáneas. Perspectivas, retos y alternativas, Bogotá: Universidad Nacional de Colombia; 2008. pp. 96-108.

48. Almeida N. Complejidad y transdisciplinariedad en el campo de la salud colectiva: evaluación de conceptos y aplicaciones. Salud Colectiva. 2006; 2: 123-46.

49. Morin E. Articular las disciplinas: la antigua y la nueva transdisciplinariedad [Trad. Houghton T]. Rev Itinerario Educativo. 2002 Ene-Dic; 14 (3040); 189-205.
50. Robledo-Martínez R, Agudelo-Calderón CA. Aproximación a la construcción teórica de la promoción de la salud. Rev Salud Pública. 2011; 13 (6): 1031-50.

51. Mantilla B. Evolución conceptual y normativa de la promoción de la salud en Colombia. Rev Univ Ind Santander Salud. 2011 Dic; 43 (3): 299-306.

52. Zemelman H. Voluntad de conocer. El sujeto y su pensamiento en el paradigma crítico. México D. F.: Antrophos; 2005.

53. Granda E. El saber en salud pública en un ámbito de pérdida de antropocentrismo y ante una visión de equilibrio ecológico. En: Granda E. La salud y la vida (Vol. 1). Quito: Ministerio de Salud Pública del Ecuador, ops, Conasa, Consejo Nacional de Educación Superior, Universidad de Cuenca, Universidad Nacional de Loja, Alames; 2009. pp. 187-224.

54. Chapela MC. Promoción de la Salud. Un instrumento de poder y una alternativa emancipatoria. En: Jarillo E, Guinsberg E, editores. Temas y desafíos en salud colectiva. Buenos Aires: Lugar; 2007. pp. 347-73.

55. Ghiso A. Prácticas generadoras de saber. Reflexiones freirianos en torno a las claves de la sistematización. Medellín: Fundación Universitaria Luis Amigó; 2006 [Internet] [acceso: 16 de julio del 2014]. Disponible en: http://virtual.funlam.edu. co/repositorio/sites/default/files/repositorioarchivos/2011/05/practicas_generadoras_saber_ponencia_aghiso_recurso_propio_unidad_3.895.pdf.

56. De Souza Minayo MC. Interdisciplinariedad y pensamiento complejo en el área de la salud. Salud Colectiva. 2008; 4 (1): 5-8.

57. Filice de Barros N. "Jamás fuimos complementarios..." Desafíos de la interdisciplinariedad en el campo de la salud. En: Martínez C, Chapela MC, Ruiz-Velasco V, coordinadores. En el juego de los espejos. Multi, inter, transdisciplina e investigación cualitativa en salud. México: Universidad Autónoma Metropolitana Xochimilco; 2013.

58. Najmanovich D. Estética del pensamiento complejo. Andamios. Revista de Investigación Social 2005 jun; 1 (2): 19- 42.

59. Ayres, J. Repensando conceptos y prácticas en salud pública. Medellín: Rev Fac Nac Salud Pública 2002; 20 (2): 67-82.

60. Sousa Santos B. Una epistemología del sur. México: Siglo XXI, Clacso; 2009. 